\title{
Estimation of the Doping Dependence of Antiferromagnetism in the Copper Oxide Material
}

\author{
Shiping Feng ${ }^{1,2}$, Yun Song ${ }^{1}$, and Zhongbing Huang ${ }^{1}$ \\ 1*Department of Physics, Beijing Normal University, Beijing 100875, China and \\ ${ }^{2}$ National Laboratory of Superconductivity, Academia Sinica, Beijing 100080, China
}

Within the $t-J$ model, we study the doping dependence of antiferromagnetism in the copper oxide materials by considering quantum fluctuations of spinons in the random-phase-approximation. The staggered magnetization vanishes around doping $\delta=5 \%$ for a reasonable parameter value $t / J=5$, which is in agreement with the experiments on copper oxide materials.

75.10.Jm, 75.10.+x, 71.45.-d

Typeset Using REVTEX 
Since the discovery that the copper oxide sheets in the High Tc superconductors show strong antiferromagnetic (AF) spin correlations [1], there has been increased interest in studying magnetic properties of these systems. This followed from the argument, made by Anderson and many other researchers [2], that the essential physics of the copper oxide superconductors is contained in doped antiferromagnets, where a central issue is the relationship between the hole doping and AF spin correlations [3,4]. In particular, the phase diagrams of these materials as functions of hole doping have been established by neutron scattering, muon spin rotation, and magnetic resonance measurements [5]. When the hole doping concentration exceeds some critical value (about 5\%), AF long-range order (AFLRO) disappears and the materials are converted into nonmagnetic metals. It is believed [2] that the physics of these materials may be effectively described by a 2D, large U Hubbard model or its equivalent, the $t-J$ model.

In an attempt to understand the relationship between the hole doping and AF spin correlations, many authors have studied holes moving in the background of the spin resonating valence bond state [2], spin flux phase [6], and spiral spin phase [7]. A hole in the AF background has a very large effective mass because of frustrations [8]. Numerical simulations suggest [9] that the 2D $t-J$ model is Néel ordered at half-filling at temperature $\mathrm{T}=0$, and the suppression of AFLRO as a consequence of hole doping has already been studied by using variational Monte-Carlo technique [10]. Recently, the fermion-spin theory has been employed to study the ground-state properties of the 2D $t-J$ model [11, 12] within the meanfield-approximation (MFA). The results show that the magnetized $\pi$-flux state, which is the coexistence of AFLRO with a $\pi$-flux state, has the lowest energy at half-filling, and the AFLRO is destroyed by hole doping of the order $\sim 10 \% \sim 15 \%$ for reasonable values of the parameters. However, quantum fluctuations are dropped completely in this mean-field treatment. In fact, there is a strong coupling between spin (spinon) and charge (holon) degrees of freedom [13]. It has been emphasized [2] that for small spin values, strong quantum fluctuations of spinons may generate a novel spin liquid ground-state. The purpose of this paper is to study these issues within the $t-J$ model by considering quantum fluctuations of 
spinons.

We start from the $t$ - $J$ model on a square lattice which can be written as

$$
H=-t \sum_{i \sigma, \eta>0} C_{i \sigma}^{\dagger} C_{i+\eta \sigma}+\text { h.c. }-\mu \sum_{i \sigma} C_{i \sigma}^{\dagger} C_{i \sigma}+J \sum_{i, \eta>0} \mathbf{S}_{i} \cdot \mathbf{S}_{i+\eta},
$$

with $\eta=\hat{x}, \hat{y}, C_{i \sigma}^{\dagger}\left(C_{i \sigma}\right)$ are the electron creation (annihilation) operators, $\mathbf{S}_{i}=C_{i}^{\dagger} \sigma C_{i} / 2$ are spin operators with $\sigma=\left(\sigma_{x}, \sigma_{y}, \sigma_{z}\right)$ as Pauli matrices, and $\mu$ is the chemical potential. The $t$ - $J$ Hamiltonian (1) is supplemented by the on-site local constraint, $\sum_{\sigma} C_{i \sigma}^{\dagger} C_{i \sigma} \leq 1$, i.e., there should be no doubly occupied sites.

The crucial requirement for the $t-J$ model is to impose this local constraint for a proper understanding of the physical properties of the copper oxide materials [14]. The local nature of the constraint is of prime importance, and its violation may lead to unphysical results [14. To avoid these difficulties, a fermion-spin transformation based on the charge-spin separation

$$
C_{i \uparrow}=h_{i}^{\dagger} S_{i}^{-}, \quad C_{i \downarrow}=h_{i}^{\dagger} S_{i}^{+},
$$

was proposed [11] to study the $t$ - $J$ model, where the spinless fermion operator $h_{i}$ keeps track of the charge (holon) while the pseudospin operator $S_{i}$ keeps track of the spin (spinon). In this case, the on-site local constraint for single occupancy is satisfied even in the MFA. For discussing quantum spin problems within the framework of fermion many particle theory, we map the quantum spin onto the spinless fermion representation via the 2D Jordan-Wigner [15] transformation, $S_{i}^{+}=f_{i}^{\dagger} e^{i \theta_{i}}, S_{i}^{-}=f_{i} e^{-i \theta_{i}}$, with $f_{i}$ as a spinless fermion, phase factor $\theta_{i}=\sum_{l \neq i} f_{l}^{\dagger} f_{l} B_{i l}$, and $B_{i l}=\operatorname{Im} \ln \left(Z_{l}-Z_{i}\right)$, in the complex representation of the lattice sites $Z_{l}=X_{l}+i Y_{l}$. In this case, it has been shown that the $t$ - $J$ model can be expressed as $11,12,16$

$$
\begin{gathered}
H=H_{0}+H_{I}^{(1)}+H_{I}^{(2)} \\
H_{0}=-\mu \sum_{i} h_{i}^{\dagger} h_{i}+\frac{J_{e f f}}{2} \sum_{i, \eta>0}\left(e^{i \int_{i}^{i+\eta} \mathbf{A} \cdot d \mathbf{l}} f_{i}^{\dagger} f_{i+\eta}+e^{-i \int_{i}^{i+\eta} \mathbf{A} \cdot d \mathbf{l}} f_{i+\eta}^{\dagger} f_{i}\right) \\
-2 J_{\text {eff }} \sum_{i} f_{i}^{\dagger} f_{i}+\frac{1}{2} N J_{\text {eff }},
\end{gathered}
$$




$$
\begin{gathered}
H_{I}^{(1)}=-t \sum_{i, \eta>0} h_{i} h_{i+\eta}^{\dagger}\left(e^{i \int_{i}^{i+\eta} \mathbf{A} \cdot d \mathbf{l}} f_{i}^{\dagger} f_{i+\eta}+e^{-i \int_{i}^{i+\eta} \mathbf{A} \cdot d \mathbf{l}} f_{i+\eta}^{\dagger} f_{i}\right)+h . c \\
H_{I}^{(2)}=\frac{1}{2} \sum_{i, \eta>0}\left(2 J_{e f f}\right) f_{i}^{\dagger} f_{i} f_{i+\eta}^{\dagger} f_{i+\eta}
\end{gathered}
$$

where $N$ is the number of sites, and $J_{\text {eff }} \approx J\left\langle\left(h_{i} h_{i}^{\dagger}\right)\left(h_{i+\eta} h_{i+\eta}^{\dagger}\right)\right\rangle, H_{I}^{(1)}$ and $H_{I}^{(2)}$ are the spinon-holon and spinon-spinon interaction Hamiltonians, respectively. $\mathbf{A}\left(r_{i}\right)$ is the vector potential created by the 2D Jordan-Wigner phase factor, which relates the $2 \mathrm{D}$ quantum spin problem to the fermion's flux phase problem [6]. The Hamiltonian (3) contains both phase and amplitude fluctuations. The phase fluctuations can be studied by using the anyon and eikonal expansion techniques [17]. However, the essential ingredients to give a qualitatively correct description of the global ground-state properties perhaps are included in amplitude fluctuations [16]. Therefore, in this paper, we only discuss the amplitude fluctuations and treat the phase factor in eq. (3) in MFA, as in the previous works [11,12,16]. For convenience, we choose the gauge field

$$
\mathbf{A}_{i j}=\frac{\pi}{2}\left(y_{j}-y_{i}\right)(-1)^{i} \hat{y}
$$

which is produced by a $\frac{1}{2}$ flux quantum (or phase $\pi$ ) through each plaquette.

For discussing the self-consistent spinon and holon Green's functions, we define the nearest-neighbor spin bond-order amplitude, Néel order parameter, and holon particle-hole parameter as

$$
\begin{gathered}
\chi=\left\langle S_{i}^{+} S_{i+\eta}^{-}\right\rangle=\left\langle e^{i \int_{i}^{i+\eta} \mathbf{A} \cdot d \mathbf{l}} f_{i}^{\dagger} f_{i+\eta}\right\rangle \\
\left\langle S_{i}^{Z}\right\rangle=(-1)^{i} M \\
\phi=\left\langle h_{i}^{\dagger} h_{i+\eta}\right\rangle
\end{gathered}
$$

respectively. This means we consider the coexistence of AFLRO with a $\pi$-flux state, and this state, referred to as the magnetized $\pi$-flux state, does not break the time-reversal symmetry and parity 11, 12,16]. The site subscripts of the order parameters $\chi, \phi$, and $M$ have been dropped since the system is translation invariant. 
Since there are two inequivalent sublattices A and B in the AF system, the one-particle spinon and holon Matsubara Green's functions can be defined as matrices,

$$
\begin{array}{r}
D\left(\mathbf{k}, \tau-\tau^{\prime}\right)=D_{L}\left(\mathbf{k}, \tau-\tau^{\prime}\right)+D_{T}\left(\mathbf{k}, \tau-\tau^{\prime}\right), \\
D_{L}\left(\mathbf{k}, \tau-\tau^{\prime}\right)=-\left(\begin{array}{cc}
\left\langle T_{\tau} f_{k}^{A}(\tau) f_{k}^{A \dagger}\left(\tau^{\prime}\right)\right\rangle & 0 \\
0 & \left\langle T_{\tau} f_{k}^{B}(\tau) f_{k}^{B \dagger}\left(\tau^{\prime}\right)\right\rangle
\end{array}\right), \\
D_{T}\left(\mathbf{k}, \tau-\tau^{\prime}\right)=-\left(\begin{array}{cc}
0 & \left\langle T_{\tau} f_{k}^{A}(\tau) f_{k}^{B \dagger}\left(\tau^{\prime}\right)\right\rangle \\
\left\langle T_{\tau} f_{k}^{B}(\tau) f_{k}^{A \dagger}\left(\tau^{\prime}\right)\right\rangle & 0
\end{array}\right),
\end{array}
$$

and

$$
\begin{array}{r}
G\left(\mathbf{k}, \tau-\tau^{\prime}\right)=G_{L}\left(\mathbf{k}, \tau-\tau^{\prime}\right)+G_{T}\left(\mathbf{k}, \tau-\tau^{\prime}\right), \\
G_{L}\left(\mathbf{k}, \tau-\tau^{\prime}\right)=-\left(\begin{array}{cc}
\left\langle T_{\tau} h_{k}^{A}(\tau) h_{k}^{A \dagger}\left(\tau^{\prime}\right)\right\rangle & 0 \\
0 & \left\langle T_{\tau} h_{k}^{B}(\tau) h_{k}^{B \dagger}\left(\tau^{\prime}\right)\right\rangle
\end{array}\right), \\
G_{T}\left(\mathbf{k}, \tau-\tau^{\prime}\right)=-\left(\begin{array}{cc}
0 & \left\langle T_{\tau} h_{k}^{A}(\tau) h_{k}^{B \dagger}\left(\tau^{\prime}\right)\right\rangle \\
\left\langle T_{\tau} h_{k}^{B}(\tau) h_{k}^{A \dagger}\left(\tau^{\prime}\right)\right\rangle & 0
\end{array}\right),
\end{array}
$$

where $\tau$ and $\tau^{\prime}$ are the imaginary times, and $\langle\ldots\rangle$ is an average over the ensemble. $D_{L}\left(G_{L}\right)$ and $D_{T}\left(G_{T}\right)$ are the longitudinal and transverse parts of the spinon (holon) Green's function $D(G)$, respectively.

A self-consistent method of treating interaction Hamiltonians as perturbation has been given in Ref. [18] within the Schwinger-boson slave-fermion theory. We [16] have used similar self-consistent calculations within the Jordan-Wigner approach to discuss the $t$ - $J$ model at half-filling and obtained some interesting results. In the following, we will generalize the method in Ref. [16] to discuss the $t-J$ model away from half-filling within the fermion-spin approach.

With the gauge choice of eq. (4), the zero-order spinon and holon Green's functions of $H_{0}$ in eq. (3b) are given by

$$
D_{L}^{(0)}(k)=\frac{i \omega_{n}+2 J_{e f f}}{\left(i \omega_{n}+2 J_{e f f}\right)^{2}-\omega_{0}^{2}(\mathbf{k})}, \quad D_{T}^{(0)}(k)=\frac{J_{e f f}\left(\sigma_{x} \cos k_{x}-\sigma_{y} \cos k_{y}\right)}{\left(i \omega_{n}+2 J_{e f f}\right)^{2}-\omega_{0}^{2}(\mathbf{k})}
$$

and

$$
G_{L}^{(0)}(k)=\frac{1}{i \omega_{n}+\mu}, \quad G_{T}^{(0)}(k)=0
$$


respectively, where $k=\left(\mathbf{k}, i \omega_{n}\right), \omega_{0}(\mathbf{k})=J_{e f f} \Gamma_{\mathbf{k}}$, and $\Gamma_{\mathbf{k}}=\sqrt{\cos ^{2} k_{x}+\cos ^{2} k_{y}}$.

According to the Dyson's theory [19], the full spinon and holon Green's functions of $H$ in eq. (3) due to considering the spinon-holon and spinon-spinon interactions are formally expressed as,

$$
\begin{gathered}
D(k)=\frac{1}{D^{(0)-1}(k)-\Sigma_{s}(k)}=\frac{1}{i \omega_{n}+2 J_{e f f}-J_{e f f}\left(\sigma_{x} \cos k_{x}-\sigma_{y} \cos k_{y}\right)-\Sigma_{s}(k)}, \\
G(k)=\frac{1}{G^{(0)-1}(k)-\Sigma_{h}(k)}=\frac{1}{i \omega_{n}+\mu-\Sigma_{h}(k)},
\end{gathered}
$$

where $\Sigma_{s}(k)$ and $\Sigma_{h}(k)$ are the spinon and holon self-energy functions, respectively. In the self-consistent calculation, the spinon and holon Green's functions cannot be self-consistently determined only by Eqs. (12) and (13), since the order parameters $\chi, M$, and $\phi$ are also included in the spinon and holon self-energy functions, and we must have another set of self-consistent equations to determine these parameters. According to the definitions of the order parameters in Eqs. (5)-(7) and the gauge choice of (4), we can get the following equations,

$$
\begin{array}{r}
\chi=\frac{1}{4} \frac{2}{N} \sum_{\mathbf{k}(r e d)} \frac{1}{\beta} \sum_{i \omega_{n}} \operatorname{Tr}\left[\left(\sigma_{x} \cos k_{x}-\sigma_{y} \cos k_{y}\right) D_{T}\left(\mathbf{k}, i \omega_{n}\right)\right], \\
M=\frac{1}{2} \frac{2}{N} \sum_{\mathbf{k}(r e d)} \frac{1}{\beta} \sum_{i \omega_{n}} \operatorname{Tr}\left[\sigma_{z} D_{L}\left(\mathbf{k}, i \omega_{n}\right)\right], \\
\phi=\frac{1}{2} \frac{2}{N} \sum_{\mathbf{k}(r e d)} \gamma_{\mathbf{k}} \frac{1}{\beta} \sum_{i \omega_{n}} \operatorname{Tr}\left[\sigma_{x} G_{T}\left(\mathbf{k}, i \omega_{n}\right)\right], \\
\delta=\frac{1}{2} \frac{2}{N} \sum_{\mathbf{k}(r e d)} \frac{1}{\beta} \sum_{i \omega_{n}} \operatorname{Tr} G_{L}\left(\mathbf{k}, i \omega_{n}\right),
\end{array}
$$

where (red) means the summation is carried only over the reduced Brillouin Zone. The order parameters $\chi, M, \phi$, and chemical potential $\mu$ can be obtained by self-consistently solving Eqs. (14)-(17).

Although the matrix analysis is quite tedious, the calculation of the first-order self-energy (Hartree-Fock self-energy) function is straightforward. Following the discussions in Ref. [16], we obtain the spinon Hartree-Fock self-energy 


$$
\Sigma_{s L}^{(H F)}(k)=2 J_{e f f}-m \rho \sigma_{z}, \quad \Sigma_{s T}^{(H F)}(k)=2\left(\phi t-\chi J_{e f f}\right)\left(\sigma_{x} \cos k_{x}-\sigma_{y} \cos k_{y}\right),
$$

and the holon Hartree-Fock self-energy

$$
\Sigma_{h L}^{(H F)}(k)=0, \quad \Sigma_{h T}^{(H F)}(k)=\epsilon_{\mathbf{k}} \sigma_{x}
$$

where $\rho=(1-2 \chi) J_{e f f}+2 t \phi, m=\frac{4 M J_{e f f}}{\rho}, \epsilon_{\mathbf{k}}=4 \chi t \gamma_{\mathbf{k}}$, and $\gamma_{\mathbf{k}}=\frac{1}{2}\left(\cos k_{x}+\cos k_{y}\right)$. $\Sigma_{s L}^{(H F)}\left(\Sigma_{h L}^{(H F)}\right)$ and $\Sigma_{s T}^{(H F)}\left(\Sigma_{h T}^{(H F)}\right)$ are the longitudinal and transverse parts of the spinon (holon) Hartree-Fock self-energy $\Sigma_{s}^{(H F)}\left(\Sigma_{h}^{(H F)}\right)$, respectively. Substituting these results of $\Sigma_{s L}^{(H F)}(k), \quad \Sigma_{s T}^{(H F)}(k), \quad \Sigma_{h L}^{(H F)}(k)$, and $\Sigma_{h T}^{(H F)}(k)$ into Eqs. (12) and (13), we obtain selfconsistent Hartree-Fock (SCHF) spinon and holon Green's functions,

$$
D_{L}^{(H F)}(k)=\frac{i \omega_{n}-m \rho \sigma_{z}}{\left(i \omega_{n}\right)^{2}-\omega^{2}(\mathbf{k})}, \quad D_{T}^{(H F)}(k)=\frac{\rho\left(\sigma_{x} \cos k_{x}-\sigma_{y} \cos k_{y}\right)}{\left(i \omega_{n}\right)^{2}-\omega^{2}(\mathbf{k})}
$$

and

$$
G_{L}^{(H F)}(k)=\frac{i \omega_{n}}{\left(i \omega_{n}\right)^{2}-\epsilon_{\mathbf{k}}^{2}}, \quad G_{T}^{(H F)}(k)=\frac{\epsilon_{\mathbf{k}} \sigma_{x}}{\left(i \omega_{n}\right)^{2}-\epsilon_{\mathbf{k}}^{2}},
$$

respectively, where $\omega(\mathbf{k})=\rho E_{\mathbf{k}}$ and $E_{\mathbf{k}}=\sqrt{\Gamma_{\mathbf{k}}^{2}+m^{2}}$. This SCHF solution is exactly the usual self-consistent MFA in the previous works [1],12]. At half-filling, the staggered magnetization and ground-state energy are $M=0.389$ and $E_{0}=-0.33 J$ per bond, respevtively. Away from half-filling, AFLRO vanishes around doping $\delta=10-15 \%$ for reasonable values of the parameter $t / J$, which are in rough agreement with experiments [5] and Monte-Carlo simulations 10. In this case, the SCHF Hamiltonian describes free spinons and holons moving in an average Chern-Simons gauge field of flux $\pi$ per plaquette [11].

The mean-field phase boundary between AFLRO and disordered states is, of course, at somewhat higher doping $\delta$ than the value given by experiments and numerical simulations. The $t$ - $J$ model is characterized by a competition between the kinetic energy $(t)$ and magnetic energy $(J)$. The magnetic energy $J$ favors an AFLRO for spins, whereas the kinetic energy $t$ favors delocalization of holes and tends to destroy AFLRO. According to the experiments [5], the suppression of AFLRO depends on both hole dopings and quantum fluctuations of spinons. Therefore in this paper the holon part will be restricted to the Hartree-Fock level, 
and we consider quantum fluctuations of spinons due to the spinon-spinon interaction $H_{I}^{(2)}$ in Eq. (3d) within the random-phase-approximation (RPA).

The analytic spinon Green's function is very complicated as emphasized in Refs. 16,18. To make the actual calculation feasible, we start from the SCHF solution, i.e., the full spinon Green's function is replaced by the spinon Hartree-Fock Green's function in the calculation of the spinon RPA self-energy [16, 18]. The detailed process to obtain the spinon RPA Green's function within the $t-J$ model at half-filling has been given in Ref. 16. Following the pocedure described there [16], we get the spinon RPA Green's function in the present case,

$$
\begin{gathered}
D^{(R P A)}(k)=\frac{1}{D^{(H F)-1}(k)-\Sigma^{(R P A)}(k)} \\
=\frac{i \omega_{n}-[m \rho-A(\mathbf{k})] \sigma_{z}+[\rho+B(\mathbf{k})]\left(\sigma_{x} \cos k_{x}-\sigma_{y} \cos k_{y}\right)}{\left(i \omega_{n}\right)^{2}-\omega_{R P A}^{2}(\mathbf{k})},
\end{gathered}
$$

where $\omega_{R P A}(\mathbf{k})=\sqrt{[m \rho-A(\mathbf{k})]^{2}+[\rho+B(\mathbf{k})]^{2} \Gamma_{\mathbf{k}}^{2}}$, and

$$
\begin{array}{r}
A(\mathbf{k})=2 J_{e f f}^{2}\left(\frac{2}{N}\right)^{2} \sum_{\mathbf{p q}(r e d)} \frac{1+\cos \left(p_{x}-p_{y}\right)}{\varepsilon_{R P A}(\mathbf{p})}\left(1-\frac{m^{2}}{E_{\mathbf{q}} E_{\mathbf{p}+\mathbf{q}}}\right) \\
\times \frac{\frac{m}{E_{\mathbf{k}+\mathbf{p}}}\left(\omega_{\mathbf{q}}+\omega_{\mathbf{p}+\mathbf{q}}+\omega_{\mathbf{k}+\mathbf{p}}\right)+\omega_{\mathbf{k}}}{\left(\omega_{\mathbf{q}}+\omega_{\mathbf{p}+\mathbf{q}}+\omega_{\mathbf{k}+\mathbf{p}}\right)^{2}+\omega_{\mathbf{k}}^{2}} \\
B(\mathbf{k})=2 J_{e f f}^{2}\left(\frac{2}{N}\right)^{2} \sum_{\mathbf{p q}(r e d)} \frac{1+\cos \left(p_{x}-p_{y}\right)}{\varepsilon_{R P A}(\mathbf{p})} \frac{\gamma_{\mathbf{p}}}{E_{\mathbf{k}+\mathbf{p}}} \frac{\Gamma_{\mathbf{q}}^{2}}{E_{\mathbf{q}} E_{\mathbf{p}+\mathbf{q}}} \\
\times \frac{\left(\omega_{\mathbf{q}}+\omega_{\mathbf{p}+\mathbf{q}}+\omega_{\mathbf{k}+\mathbf{p}}\right)+\omega_{\mathbf{k}}}{\left(\omega_{\mathbf{q}}+\omega_{\mathbf{p}+\mathbf{q}}+\omega_{\mathbf{k}+\mathbf{p}}\right)^{2}+\omega_{\mathbf{k}}^{2}} .
\end{array}
$$

Here $\varepsilon_{R P A}(p)$ is the spinon RPA dielectric function. With the above holon and spinon Green's functions, we obtain the self-consistent equations at zero temperature ,

$$
\begin{array}{r}
\chi=-\frac{1}{4} \frac{2}{N} \sum_{\mathbf{k}(r e d)} \frac{[\rho+B(\mathbf{k})] \Gamma_{\mathbf{k}}^{2}}{\omega_{R P A}(\mathbf{k})}, \\
M=\frac{2}{N} \sum_{\mathbf{k}(r e d)} \frac{m \rho-A(\mathbf{k})}{2 \omega_{R P A}(\mathbf{k})}, \\
\phi=\frac{2}{N} \sum_{\mathbf{k}(r e d)} \frac{\gamma_{\mathbf{k}}}{2}\left[\theta\left(\mu-\epsilon_{\mathbf{k}}\right)-\theta\left(\mu+\epsilon_{\mathbf{k}}\right)\right], \\
\delta=\frac{2}{N} \sum_{\mathbf{k}(r e d)} \frac{1}{2}\left[\theta\left(\mu-\epsilon_{\mathbf{k}}\right)+\theta\left(\mu+\epsilon_{\mathbf{k}}\right)\right],
\end{array}
$$


where the effect of quantum fluctuations of spinons from the spinon-spinon interaction have been included in $A(\mathbf{k}), B(\mathbf{k})$, and $\omega_{R P A}(\mathbf{k})$, which will affect the parameters $\chi, M$, and $\mu$, but not the parameter $\phi$ at low dopings. This is because the effect due to the variance of parameter $\chi$ in $\epsilon_{\mathbf{k}}=4 \chi t \gamma_{\mathbf{k}}$ in Eqs. (26) and (27) can be incorporated into the parameter $t / J$. On the other hand, we have shown in Ref. [11] that at low dopings the holon particle-hole order parameter $\phi$ increases roughly linearly upon doping, and is almost independent of $t / J$. Therefore in this case we can take the mean-field values of the parameter $\phi$ to calculate the RPA self-consistent equations (24) and (25) for the parameters $\chi$ and $M$. We have performed such RPA calculation. At half-filling, the result is the same as those in Ref. [16], i.e. the staggered magnetization and RPA ground-state energy are $M=0.327$ and $E_{0}=-0.332 J$ per bond, respectively, which are very close to the best numerical result [20] $M=0.31$ and $E_{0}=-0.3346 J$. Away from half-filling, AFLRO is quickly destroyed by doping of the order of $\delta=5 \%$ for a reasonable parameter value $t / J=5$, which is plotted in Fig. 1 (solid triangles). For comparison, the experimental measurements on $\left(L a_{1-x} B a_{x}\right)_{2} C u O_{4}$ (solid squares, the dashed line is guide for eyes) [5] and numerical results (solid circles) [10] are also plotted in Fig. 1. From Fig. 1, it is obvious that our result is in agreement with the experiments [5] and Monte-Carlo simulations [10]. As compared with the mean-field result [11], the present RPA results indicate that both hole dopings and quantum fluctuations of spinons lead to a strong suppression of AFLRO.

In summary, we have studied the doping dependence of AFLRO in the copper oxdie materials within the $t-J$ model. We have performed a self-consistent calculation, where the holon part is restricted to the Hartree-Fock level, and the quantum fluctuation of spinons is considered within the RPA. We have considered only the amplitude fluctuations. AFLRO vanishes around doping $\delta=5 \%$ for a reasonable parameter value $t / J=5$, which is in agreement with the experiments on copper oxide materials [5] and Monte-Carlo simulations [10]. 


\section{ACKNOWLEDGMENTS}

The authors would like to thank Prof. X. Xu and Prof. Z. X. Zhao for helpful discussions. This work is supported by the National Science Foundation Grant No. 19474007 and the

Trans-Century Training Programme Foundation for the Talents by the State Education Commission of China. 


\section{REFERENCES}

* $\quad$ Mailing address.

[1] D. Vaknin et al., Phys. Rev. Lett. 58, 2802 (1987).

[2] P. W. Anderson, Science 235, 1196 (1987); F. C. Zhang and T. M. Rice, Phys. Rev. B37, 3759 (1988).

[3] A. P. Kampf, Phys. Rep. 249, 219 (1994), and references therein.

[4] See, e.g., "High Temperature Superconductivity", Proc. Los Alamos Symp., 1989, K. S. Bedell et al., eds. (Addison-Wesley, Redwood City, California, 1990).

[5] Y. Kitaoka et al., Physica C153-155, 733 (1988); J. H. Brewer et al., Phys. Rev. Lett. 60, 1073 (1988); D. W. Cooke et al., Phys. Rev. B52, R3864 (1995).

[6] I. Affleck and J. B. Marston, Phys. Rev. B 37, 3774 (1988).

[7] B. Shraiman and E. Siggia, Phys. Rev. Lett. 62, 1564 (1989).

[8] C. L. Kane, P. A. Lee, and N. Read, Phys. Rev. B 39, 6880 (1989); Z. B. Su, Y. M. Li, W. Y. Lai, and L. Yu, Phys. Rev. Lett. 63, 1318 (1989).

[9] S. Liang, B. Doucot, and P. W. Anderson, Phys. Rev. Lett. 61, 365 (1988).

[10] T. K. Lee and Shiping Feng, Phys. Rev. B 38, 11809 (1988).

[11] Shiping Feng, Z. B. Su, and L. Yu, Phys. Rev. B 49, 2368 (1994); Mod. Phys. Lett. B7, $1013(1993)$.

[12] Shiping Feng, Physica C232, 119 (1994); X. Xu, Y. Song, and Shiping Feng, Mod. Phys. Lett. B9, 1623 (1995); X. Xu, Y. Song, and Shiping Feng, Acta Physica Sinica, 45, 1390 (1996).

[13] See, e. g., the review, L. Yu, in "Recent Progress in Many-Body Theories", vol. 3, T. L. Ainsworth et al., eds.( Plenum, 1992) P.157. 
[14] Shiping Feng, J. B. Wu, Z. B. Su, and L. Yu, Phys. Rev. B47, 15192 (1993); L. Zhang, J. K. Jain, and V. J. Emery, Phys. Rev. B47, 3368 (1993).

[15] E. Mele, Phys. Scripta T27, 82 (1988); E. Fradkin, Phys. Rev. Lett. 63, 322 (1989); G. Baskaran, in "Two-Dimensional Strongly Correlated Electronic Systems", Z. Z. Gan and Z. B. Su, eds.(Gordon and Breach), 1989, p.72; Y. R. Wang, Phys. Rev. B 46, 151 (1992).

[16] Shiping Feng, Phys. Rev. B53, 11671 (1996).

[17] A. M. Tikofsky and R. B. Laughlin, Phys. Rev. B 50, 10165 (1994); D. V. Khveshchenko and P. C. E. Stamp, Phys. Rev. Lett. 71, 2118 (1993).

[18] Y. M. Li et al., Phys. Rev. B 45, 5428 (1992).

[19] G. D. Mahan, Many-Particle Physics (Plenum, New York, 1981).

[20] N. Trivedi and D. M. Ceperley, Phys. Rev. B 40, 2747 (1989). 


\section{FIGURES}

FIG. 1. Calculated values of the staggered magnetization of the $t-J$ model as a function of the hole concentration $\delta$ for $t / J=5$ (solid triangles). The solid squares and solid circles correspond to the experimental measurements on $\left(L a_{1-x} \mathrm{Ba}_{x}\right)_{2} \mathrm{CuO} \mathrm{O}_{4}$ [5] and numerical results [10], respectively. $M_{0}$ is the value of the staggered magnetization at half-filling. 